\title{
Problems in the evaluation of polyvinylpyrrolidone (PVP) elution from polysulfone membrane dialyzers sterilized by gamma-ray irradiation
}

\author{
Jun Murakami ${ }^{1}$, Iwakazu Kaneko ${ }^{1}$, Naoki Kimata², Michio Mineshima ${ }^{1 *}$ and Takashi Akiba
}

\begin{abstract}
Background: Adverse reactions, such as anaphylactoid shock, have been reported to occur frequently with the use of polysulfone (PSf) membrane dialyzers. Polyvinylpyrrolidone (PVP) elution from the membrane may be a key factor in these reactions. In this paper, we discuss the problems in the evaluation of PVP elution from PSf membrane dialyzers sterilized by gamma-ray irradiation.

Methods and Results: PVP concentrations in the filling solutions in some wet-type PSf membrane dialyzers are measured by Müller's method as a standard measurement method. The PVP concentrations in autoclave (AC)-sterilized dialyzers were one order of magnitude higher than those in the solutions in dialyzers sterilized by gamma-ray irradiation. Because it is difficult to determine the PVP concentrations in the filling solutions sterilized by gamma-ray irradiation by Müller's method, single-fractionated-component PVP solutions with fractionated components of PVP (K90 or K30) were prepared and the PVP concentrations of the solutions before and after gamma-ray irradiation were determined. The results indicated that the PVP concentrations in the solutions could be determined by Müller's method before irradiation, whereas PVP was undetectable in the solution after irradiation. For single-fractionated-component PVP solutions with K90 and K30, the changes in the structure of PVP before and after gamma-ray irradiation were analyzed by high-performance liquid chromatography (HPLC). The single-fractionated-component PVP solutions with K90 and K30 had a broad peak at retention times of 15 and 19 min, respectively, prior to the gamma-ray irradiation, whereas both solutions showed a similar sharp peak at a retention time of 23 min after the irradiation. Based on these results, it is surmised that PVP is degraded by irradiation to yield PVP degradation products of low molecular weight.

Furthermore, nuclear magnetic resonance (NMR) spectroscopy for PVP K90 solution was performed to confirm the signals from the vinylpyrrolidone (VP) skeletal structures. Signals from the VP skeletal structures were detected before the gamma-ray irradiation but disappeared after the irradiation. Thus, it appears that the degradation products of PVP without VP skeletal structures cannot be detected by Müller's method.
\end{abstract}

Conclusions: The measurement of PVP concentrations by Müller's method is inappropriate for the evaluation of PVP elution from PSf membrane dialyzers sterilized by gamma-ray irradiation.

Keywords: Polysulfone membrane dialyzer, Gamma-ray-irradiated sterilization, PVP, PVP elution, Müller's method

\footnotetext{
*Correspondence: mmine@twmu.ac.jp

'Department of Clinical Engineering, Tokyo Women's Medical University, 8-1

Kawada, Shinjuku-ku, Tokyo 162-8666, Japan

Full list of author information is available at the end of the article
} 


\section{Background}

Currently, polysulfone (PSf) membrane dialyzers are used worldwide because of their excellent solute-removal capability and beneficial cost-performance ratio. However, adverse reactions induced by PSf membranes, including relatively mild reactions and severe adverse reactions such as anaphylactoid shock, have been reported [1-3]. Polyvinylpyrrolidone (PVP) is usually used in PSf membranes as a hydrophilic and a pore-opening agent. While there have been a few reports identifying the eluted PVP from PSf membranes as the cause of severe anaphylactoid shock, most reported studies have indicated that the causative factor is unclear. Some studies have reported differences in the frequency of appearance of allergy symptoms in the same patient undergoing hemodialysis with the same type of PSf membrane dialyzers but sterilized by different methods [4, 5].

Based on the above background, we focused on the elution of PVP or PVP degradation products from PSf membranes sterilized by different methods. In this paper, we discuss the problems in the evaluation of PVP elution from PSf membrane dialyzers sterilized by gamma-ray irradiation.

\section{Methods}

We carried out an in vitro study consisting of four experiments to accomplish our study objective.

1. Analysis of the filling solutions on the blood and dialysate sides of the dialyzers

The filling solutions on both the blood and dialysate sides in several wet-type membrane dialyzers were sampled and analyzed. The dialyzers used for this experiment consisted of five types of PSf membranes: RENAK PS-1.6, RENAK PS-1.6y (a trial gamma-ray-irradiated dialyzer), APS-15SA, TS-1.6UL, and VPS-15HA. FDY-150GW with polyether polymer alloy (PEPA) membrane and BG-1.6PQ with polymethylmethacrylate (PMMA) membrane were also used in comparison with PSf membrane. All the dialyzers were used within 1 year of sterilization (3-11 months).

The filling solutions from the blood and dialysate side were collected at the outlet portion of the dialyzers after the inlet portion was opened. The ultraviolet (UV) absorbance at the wavelength $(\lambda)$ of $220 \mathrm{~nm}$ of each solution was measured. The PVP concentration of each solution was also measured by "Müller's method" based on iodometry [6]. The ultraviolet-visible spectrophotometer, UV-1800 (Shimadzu Corporation), was used for the ultraviolet (UV) light absorbance measurements.
2. Determination of the PVP concentration changes in single-fractionated-component PVP solutions before and after gamma-ray irradiation Single-fractionated-component PVP solutions with PVP concentrations of 10 and 50 ppm were prepared by dissolving PVP reagents (K90 or K30) in pure water and pouring them into containers. The containers, made of glass, polypropylene (PP) and polystyrene (PS), were used in order to evaluate the influence of the housing material of the dialyzer. The solutions were irradiated with gamma rays at 25 kGy. The PVP concentrations in each solution before and after the gamma-ray irradiation were measured by Müller's method and compared. The PVP reagents used were PVP K90 and K30 (Wako Pure Chemical Industries, Ltd.). The $K$ value of PVP denotes the viscosity characteristic value, which is correlated with the molecular weight. The $K$ values can be calculated from the following equation:

$$
\begin{aligned}
& K=\left(1.5 \log v_{\mathrm{rel}}-1\right) /(0.15+0.003 c) \\
& \quad+\left(300 c \log v_{\mathrm{rel}}+\left(c+1.5 c \log v_{\mathrm{rel}}\right)^{2}\right)^{1 / 2} \\
& \quad /\left(0.15 c+0.003 c^{2}\right)
\end{aligned}
$$

where $v_{\text {rel }}$ and $c$ denote the viscosity relative to water and the PVP concentration (\%) in the PVP solution, respectively.

The average molecular weights of $\mathrm{K} 90$ and K30 are 360,000 and 40,000, respectively.

3. Determination of changes of the PVP molecular weight distribution in single-fractionated-component PVP solutions before and after gamma-ray irradiation Single-fractionated-component PVP solutions with K90 or K30 with PVP concentrations of 50 ppm were prepared by dissolving PVP reagent in pure water and poured into containers made of glass. The molecular weight distributions of PVP before and after the gamma-ray irradiation were analyzed by high-performance liquid chromatography (HPLC). LaChrom Elite of Hitachi High-Technologies Corporation, with the column, Shodex Asahipak GF-HQ, of Showa Denko K.K. was used for the HPLC, with the absorbances read at the $\lambda$ wavelength of $220 \mathrm{~nm} ; 50 \mathrm{mmol} / \mathrm{L}$ of $\mathrm{NaCl}$ was used as the mobile phase solution.

4. Determination of the PVP structure changes in single-fractionated-component PVP solution before and after gamma-ray irradiation A 50-ppm solution of PVP K90 was analyzed by nuclear magnetic resonance (NMR) spectroscopy 
before and after gamma-ray irradiation to confirm the signals from the vinylpyrrolidone (VP) skeletal structures. The Avance 500-MHz spectrometer equipped with a cryoprobe (Bruker BioSpin K.K.) was used for the NMR spectroscopy. The nuclide and solvent used were hydrogen $\left({ }^{1} \mathrm{H}\right)$ and heavy water, respectively.

\section{Results}

Analysis of the filling solutions on the blood and dialysate sides of the dialyzers

Figure 1 shows the UV absorbance of the filling solutions of the dialyzers. For the PSf membrane dialyzers, the filling solutions on the dialysate side showed higher UV absorbance values than the filling solutions on the blood side. No such results were obtained for the PEPA and PMMA membrane dialyzers.

Figure 2 shows the PVP concentrations of the filling solutions measured by Müller's method. The RENAK PS-1.6 sterilized by autoclave (AC) showed a one order of magnitude higher filling solution PVP concentration than the dialyzers sterilized by gamma-ray irradiation. A significant difference in the filling solution PVP concentration was found between the

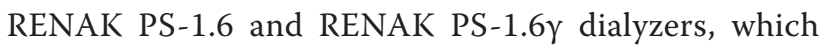

were the same type of dialyzer differing only in the sterilization method.

\section{Determination of the PVP concentration changes in single-fractionated-component PVP solutions before and after gamma-ray irradiation}

Figure 3 shows the PVP concentrations of singlefractionated-component PVP solutions measured by Müller's method before and after gamma-ray irradiation. PVP was not detectable in any of the solutions in any container that was sterilized by gamma-ray irradiation.

\section{Determination of changes of the PVP molecular weight distribution in single-fractionated-component PVP solutions before and after gamma-ray irradiation}

Figure 4 shows the HPLC results before and after gamma-ray irradiation. The peak intensities for singlefractionated-component PVP solutions with K90 and K30 before gamma-ray irradiation were observed at retention times of 15 and $19 \mathrm{~min}$, respectively. These peaks were relatively broad, suggestive of a wide distribution of molecular weights. On the other hand, after the gamma-ray irradiation, both solutions showed similar peak intensities at a retention time of $23 \mathrm{~min}$, and the peaks were relatively sharp for both.

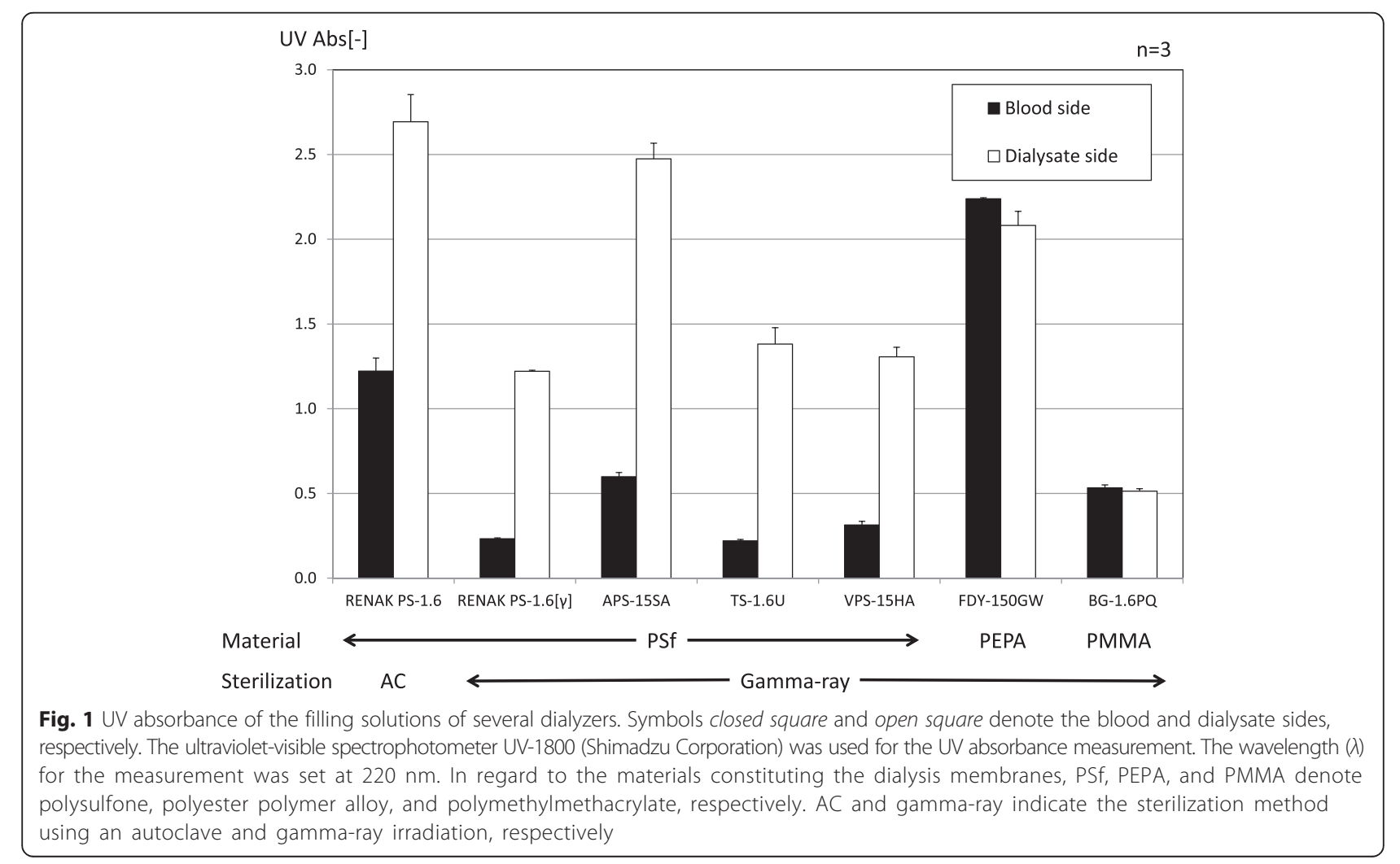




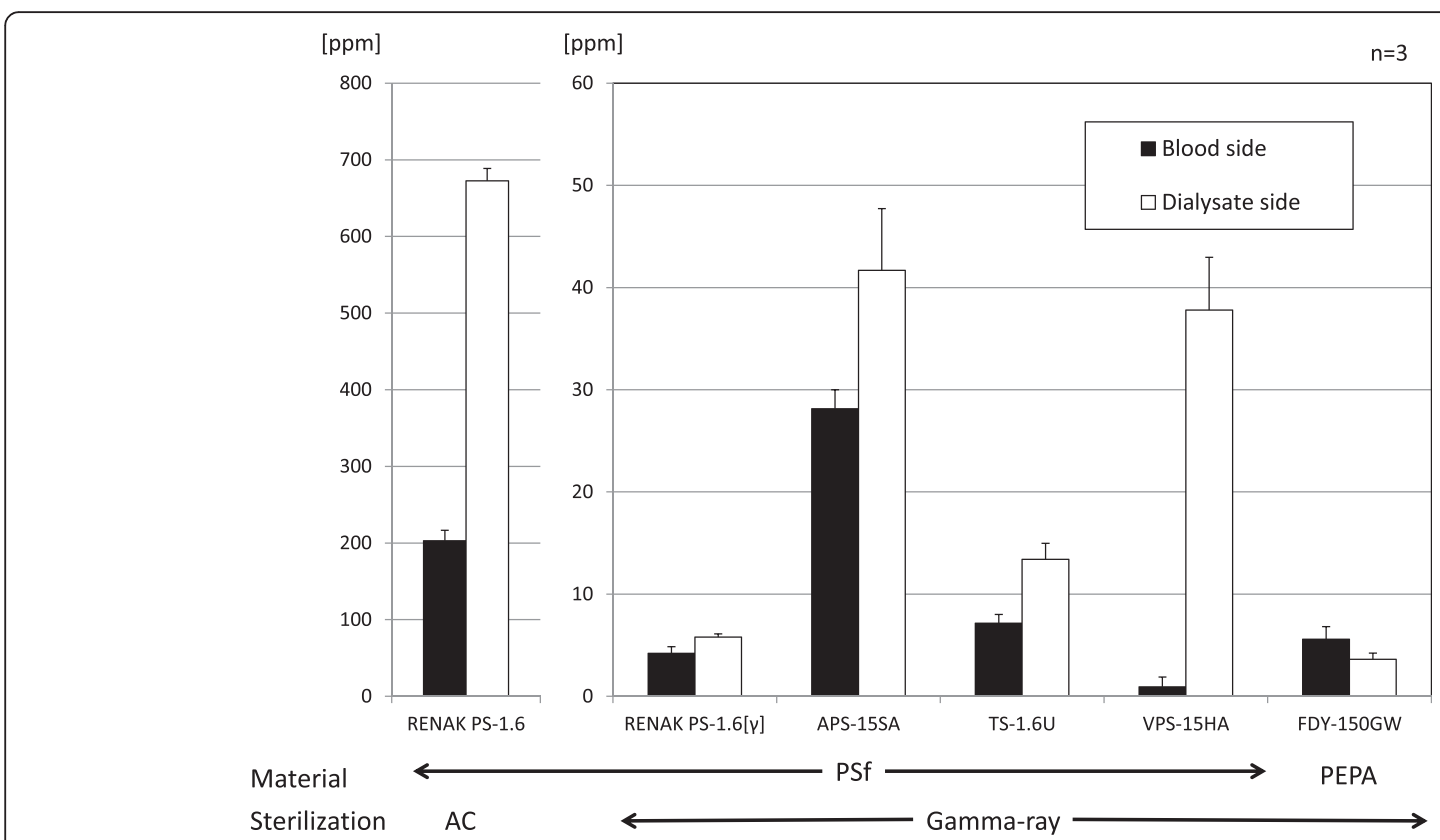

Fig. 2 PVP concentrations of the filling solutions of several dialyzers measured by Müller's method. Symbols closed square and open square denote the blood and dialysate sides, respectively. The PVP concentrations were measured by Müller's method. In regard to the materials constituting the dialysis membranes, PSf, PEPA, and PMMA denote polysulfone, polyester polymer alloy, and polymethylmethacrylate, respectively. AC and gamma-ray indicate the sterilization method using an autoclave and gamma-ray irradiation, respectively

Determination of the PVP structure changes in singlefractionated-component PVP solution before and after gamma-ray irradiation

Figure 5 shows the signals from the VP skeletal structures obtained by NMR spectroscopy. The findings confirmed changes of the PVP structure. Before the gamma-ray irradiation, the single-fractionated-component PVP solution with K90 showed clear peaks (a-e) corresponding to the VP skeletal structures. On the other hand, no such peaks were observed after the gamma-ray irradiation.

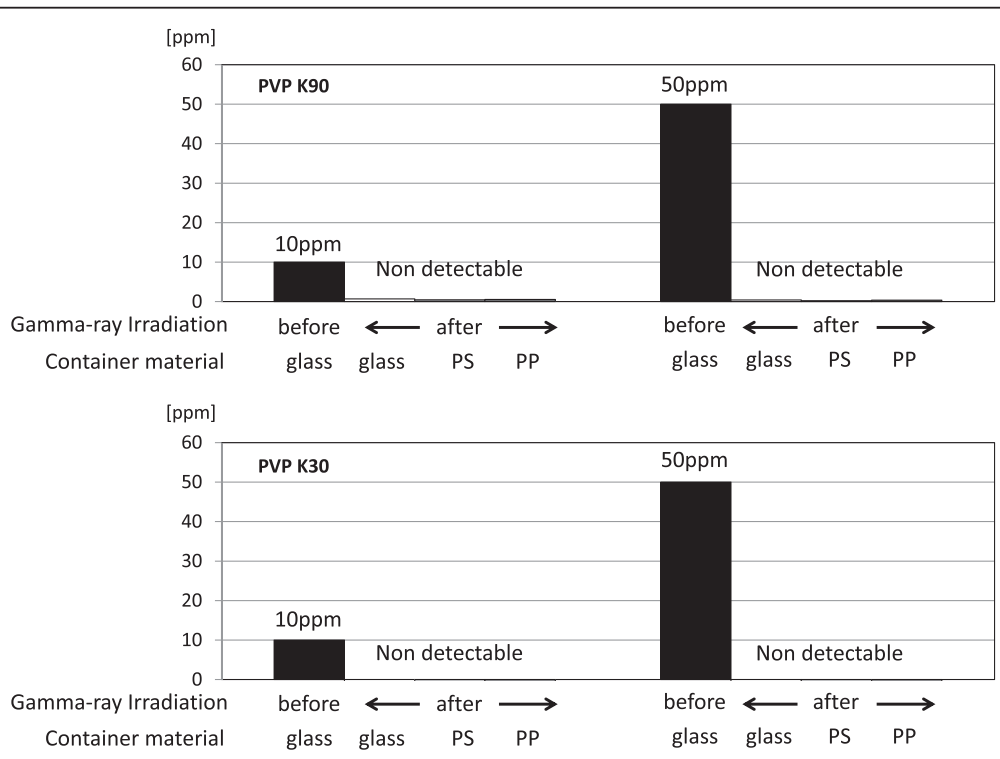

Fig. 3 PVP concentrations of single-fractionated-component PVP solutions before and after gamma-ray irradiation. PVP concentrations were measured by Müller's method. The average molecular weights of $K 90$ and K30 are 360,000 and 40,000, respectively. The containers were made of glass, polystyrene (PS), and polypropylene (PP). The initial PVP concentrations before the irradiation were set as 10 and 50 ppm. Gamma-ray irradiation was carried out at $25 \mathrm{kGy}$ 

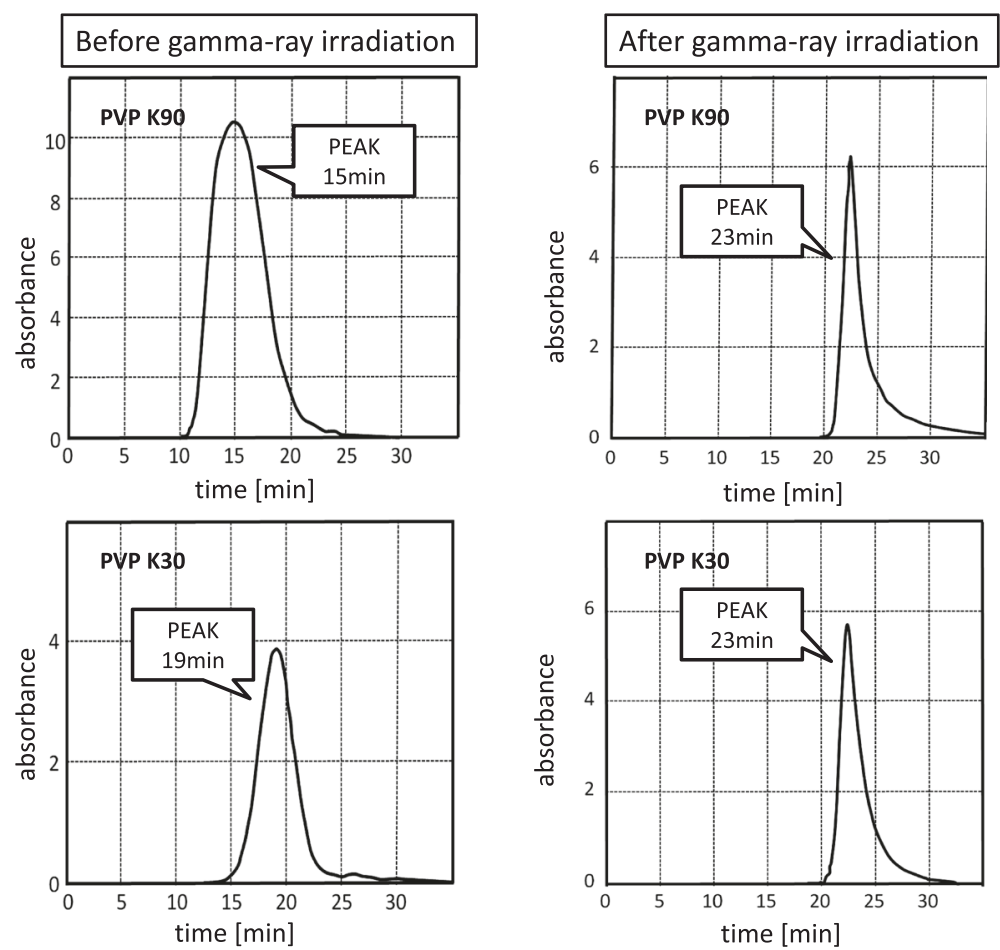

Fig. 4 PVP molecular weight distribution before and after gamma-ray irradiation determined by high-performance liquid chromatography (HPLC). The initial PVP K90 or K30 concentrations before irradiation were set at 50 ppm. LaChrom Elite of Hitachi High-Technologies Corporation with the Shodex Asahipak GF-HQ column of Showa Denko K.K. was used for the HPLC, with the measurement of the absorbance carried out at the wavelength ( $\lambda$ ) of $220 \mathrm{~nm} ; 50 \mathrm{mmol} / \mathrm{L}$ of $\mathrm{NaCl}$ was used as the mobile phase solution

\section{Discussion}

Analysis of the filling solutions on the blood and dialysate sides of the dialyzers

The differences in UV absorbance values observed between the blood- and dialysate-side filling solutions for several dialyzers seem to strongly depend on the membrane structure. PSf is one of the asymmetrical membranes and has a skin layer on the inner surface of the hollow fiber and a support layer with a gradient or porous structure. Elution from the membrane to

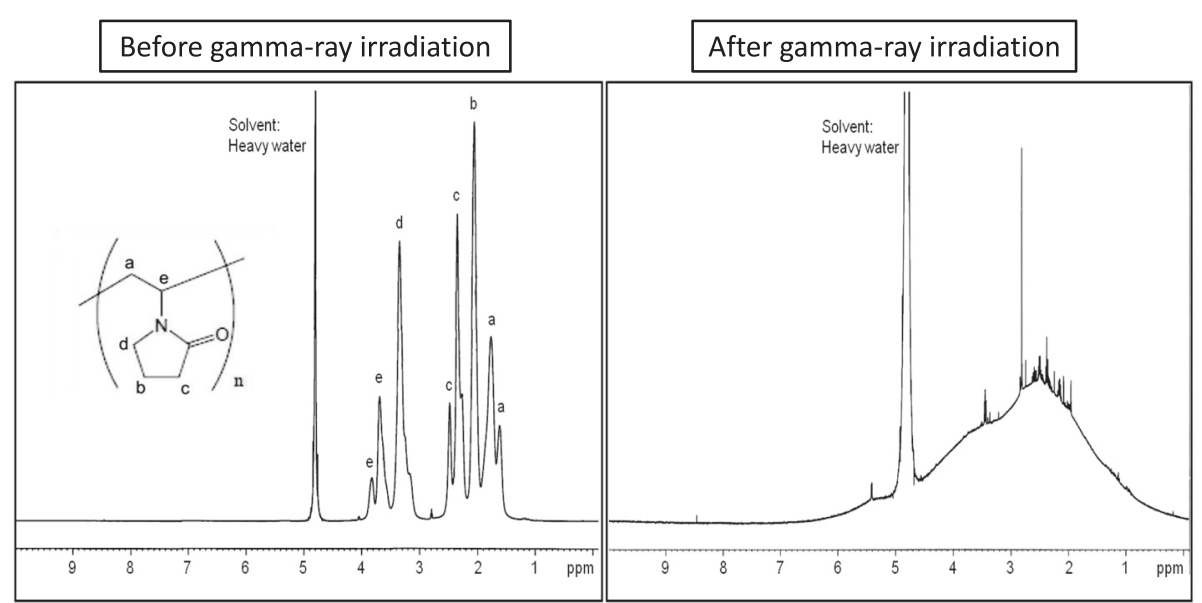

Equipment: Avance 500-MHz spectrometer equipped with a cryoprobe (Bruker Biospin K.K.) Nuclide: Hydrogen $\left({ }^{1} \mathrm{H}\right)$, Solvent: Heavy water

Fig. 5 Confirmation of signals from the vinylpyrrolidone (VP) skeletal structures by nuclear magnetic resonance (NMR) spectroscopy. The Avance 500-MHz spectrometer equipped with a cryoprobe (Bruker BioSpin K.K.) was used for the NMR instrument. The nuclide and solvent used were hydrogen $\left({ }^{1} \mathrm{H}\right)$ and heavy water, respectively 
the filling solution on the blood side is strongly restricted by the skin layer as compared to elution on the side of the dialysate. PEPA is also an asymmetrical membrane, with two skin layers on the inner and outer surfaces of the hollow fiber and a gradient/porous structure between the skin layers. PMMA is one of the symmetrical homogeneous membranes. In both PEPA and PMMA, no significant differences in elution were observed between the blood and dialysate sides, evidently because of their membrane structures.

As shown in Fig. 2, there were some differences of the PVP concentrations in the filling solutions among dialyzers sterilized by gamma-ray irradiation. Especially, VPS-15HA with PSf coated by vitamin E showed a low PVP concentration of the filling solution on the blood side as compared to the results for other dialyzers, presumably because the vitamin E coating restricts PVP elution.

There were significant differences in the filling solution PVP concentrations between the AC-sterilized and gamma-ray-sterilized dialyzers. As shown in Figs. 1 and 2, the differences in the PVP concentrations between the RENAK PS-1.6 and RENAK PS-1.6y dialyzers, which differed only in respect of the sterilization method, were greater than the differences of the UV absorbance between the dialyzers. It is thought that some substances produced after gammaray irradiation might not be detectable by Müller's method.

We can consider two reasons for these results.

First, in dialyzers sterilized by gamma-ray irradiation, the PVP elution may be influenced by cross-linkinginsolubilized PVP induced by gamma-ray irradiation.

Second, it seemed that after gamma-ray irradiation, PVP was degraded, with the production of PVP degradation products. These degradation products might not be detectable by Müller's method.

In this study, the subsequent experiments were carried out to confirm whether the latter postulation is correct or not.

\section{Analysis of PVP concentration changes in single- fractionated-component PVP solutions before and after gamma-ray irradiation}

As shown in Fig. 3, it was revealed that neither PVP nor PVP degradation products could be detected after gamma-ray irradiation in any of the containers by Müller's method. Therefore, it is thought that the use of Müller's method is inappropriate for the evaluation of PVP elution from PSf membranes sterilized by gamma-ray irradiation. Furthermore, the determination of the PVP concentration by "Müller's method" is independent of the container material.
Analysis of changes of the PVP molecular weight distribution in single-fractionated-component PVP solutions before and after gamma-ray irradiation The experiments were carried out to determine the changes in PVP induced by gamma-ray irradiation.

As shown in Fig. 4, it seemed that the structures of both PVP K90 and K30 changed after gamma-ray irradiation, with the production of PVP degradation products of low molecular weight. Both PVP K90 and K30 appeared to be depolymerized by gamma-ray irradiation, with the production of VP monomers that are thought to be poisonous.

\section{Determination of the PVP structure changes in single- fractionated-component PVP solution before and after gamma-ray irradiation}

As shown in Fig. 5, no VP skeletal structures could be detected by NMR spectroscopy in the single-fractionatedcomponent PVP solution with K90 after gamma-ray irradiation. This finding suggests that the PVP in the solution was degraded and changed to other substances and that this is the reason for the lack of detectability of the substance by Müller's method.

If it is assumed that the anaphylactoid reactions reported in patients undergoing dialysis with gammaray-irradiated PSf membrane dialyzers are caused by the degradation products of PVP produced by gammaray irradiation, it may be easy to understand why these patients test negative for PVP in the scratch and prick tests. This may also explain why patients in whom the dialyzer was changed from an autoclaved PSf membrane to a gamma-ray-irradiated PSf membrane exhibited anaphylactoid shock $[4,5]$. However, the precise relationships between such adverse reactions and the degradation products of PVP still remain unclear and the available evidence remains insufficient, and further studies are needed.

\section{Conclusions}

We demonstrated that the degradation products of PVP produced by gamma-ray irradiation lack in VP skeletal structures and cannot be detected by Müller's method. Therefore, use of Müller's method may be inappropriate for the evaluation of PVP elution from polysulfone membranes in dialyzers sterilized by gamma-ray irradiation.

\section{Competing interests \\ The authors declare that they have no competing interests.}

\section{Authors' contributions}

JM carried out the planned and performed the whole study, and created the manuscript. IK participated the experiments of the study. NK participated the discussion for the results of the experiments. MM participated the

discussions for the results of the experiments and, revised and submitted the manuscript. TA advised the study design and revised the manuscript. All authors read and approved the final manuscript. 


\section{Acknowledgements}

In this study, we utilized various instruments in the research facilities of Kawasumi Laboratories, Inc. We are grateful to Mr. Nobuyuki Hosoi of the company for his technical and accurate advice on the operation of the instruments and for clarifying our understanding of the results of the measurements.

\section{Author details}

'Department of Clinical Engineering, Tokyo Women's Medical University, 8-1 Kawada, Shinjuku-ku, Tokyo 162-8666, Japan. ${ }^{2}$ Department of Blood Purification, Kidney Center, Tokyo Women's Medical University, 8-1 Kawada, Shinjuku-ku Tokyo, 162-8666, Japan.

Received: 10 August 2015 Accepted: 6 May 2016

Published online: 20 June 2016

\section{References}

1. Arenas MD, Gil MT, Carretón MA, Moledous A, Albiach B. Adverse reactions to polysulphone membrane dialyzers during hemodialysis. Nefrologia. 2007; 27(5):638-42.

2. Huang $W H$, Lee $Y Y$, Shih LC. Delayed near-fatal anaphylactic reaction induced by the F10-HPS polysulphone haemodialyser. Nephrol Dial Transplant. 2008:23(1):423-4.

3. Bacelar Marques ID, Pinheiro KF, de Freitas do Carmo LP, Costa MC, Abensur $\mathrm{H}$. Anaphylactic reaction induced by a polysulfone/polyvinylpyrrolidone membrane in the 10th session of hemodialysis with the same dialyzer. Hemodial Int. 2011:15(3):399-403.

4. Heegard KD, Tilley MA, Stewart IJ, Edgecombe HP, Lundy JB, Renz EM, et al. Anaphylactoid reaction during first hemofiltration with a PUREMA polysulfone membrane. Int J Artif Organs. 2013;36(5):363-6.

5. Ohashi N, Yonemura K, Goto T, Suzuki H, Fujigaki Y, Yamamoto T, et al. A case of anaphylactoid shock induced by the BS polysulfone hemodialyzer but not by the F8-HPS polysulfone hemodialyzer. Clin Nephrol. 2003;60(3):214-7.

6. Müller K. Detection and determination of polyvinylpyrrolidone (PVP) and determination of active components in PVP-containing drug preparations. Pharm Acta Helv. 1968;43:107-22 (in German).

\section{Submit your next manuscript to BioMed Central} and we will help you at every step:

- We accept pre-submission inquiries

- Our selector tool helps you to find the most relevant journal

- We provide round the clock customer support

- Convenient online submission

- Thorough peer review

- Inclusion in PubMed and all major indexing services

- Maximum visibility for your research

Submit your manuscript at www.biomedcentral.com/submit 